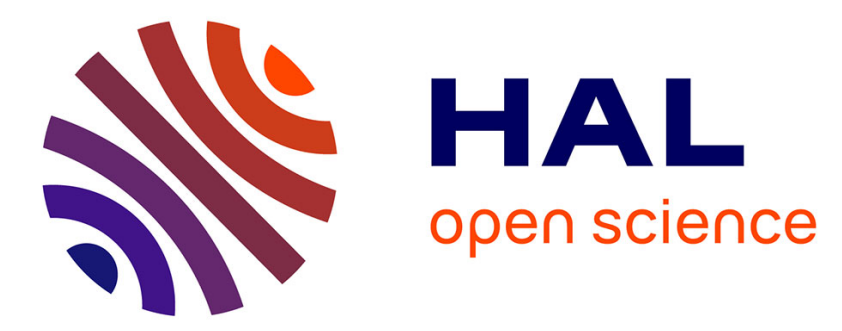

\title{
On the non-paraxial modes of two-dimensional nearly concentric resonators
}

\author{
F. Zomer, V. Soskov, A. Variola
}

\section{To cite this version:}

F. Zomer, V. Soskov, A. Variola. On the non-paraxial modes of two-dimensional nearly concentric resonators. Applied optics, 2007, 46, pp.6859-6866. in2p3-00160628

\section{HAL Id: in2p3-00160628 https://hal.in2p3.fr/in2p3-00160628}

Submitted on 6 Jul 2007

HAL is a multi-disciplinary open access archive for the deposit and dissemination of scientific research documents, whether they are published or not. The documents may come from teaching and research institutions in France or abroad, or from public or private research centers.
L'archive ouverte pluridisciplinaire HAL, est destinée au dépôt et à la diffusion de documents scientifiques de niveau recherche, publiés ou non, émanant des établissements d'enseignement et de recherche français ou étrangers, des laboratoires publics ou privés. 
LAL 07-47

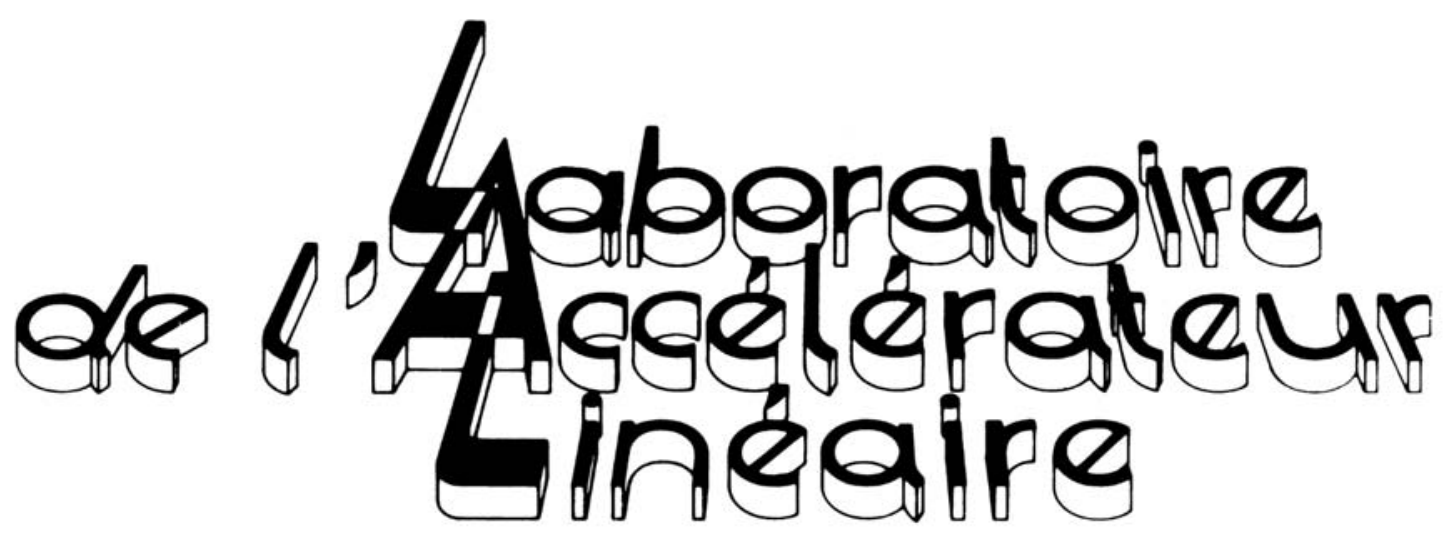

On the non-paraxial modes of two-dimensional nearly concentric resonators

F. Zomer, V. Soskov and A. Variola

LAL, Univ Paris-Sud, IN2P3/CNRS, Orsay, France

to be published in "Applied Optics"

\section{U.M.R}

de

l'Université Paris-Sud

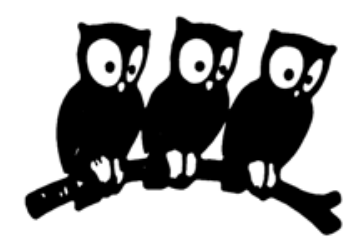

Institut National de Physique Nucléaire et de Physique des Particules du CNRS 
LAL 07-47

May 2007

\title{
On the non-paraxial modes of two-dimensional nearly concentric resonators
}

\author{
F. Zomer, V. Soskov and A. Variola \\ LAL, Univ Paris-Sud, IN2P3/CNRS, Orsay, France
}

\begin{abstract}
A non-paraxial scalar diffraction integral is used to determine numerically the resonance modes of a two dimensional nearly concentric Fabry-Perot resonator. Numerical examples are provided and results are compared to those published by Laabs and Friberg [IEEE J. Quant. El., vol. 35, pp. 198-207, 1999]. Discrepancies are reported and further discussed on the basis of the difference between the solution space supported by the numerical method used in the present work and the one used by Laabs and Friberg.
\end{abstract}




\section{INTRODUCTION}

An optical resonator made of two identical mirrors such that the distance between those two mirrors is close to twice the mirror radius of curvature is called a nearly concentric resonator. Inside such a resonator the laser beam waist can be very small, of the order of a few wavelengths 1 .

In the field of High Energy Physics, there is at present an interest for nearly concentric resonators. Firstly to increase the production rate of $\mathrm{X}$ or gamma rays by Compton scattering of a laser beam on an electron beam (in this case the cavity is located around the electron-laser interaction point). The resulting bright and highly collimated scattered X/gamma rays beams can be used for applied science applications 2, or to produce a polarized positron beams for the next generation of linear collider 3,4. Secondly, it has been proposed to measure the vacuum birefringence via photonphoton interaction thanks to two pulsed laser circulating in two concentric resonators 5. For both applications, one needs a strongly focused pulse laser beam with of high energy $(>100 \mathrm{~mJ})$ and high repetition rate $(50-350 \mathrm{MHz})$.

In the paraxial approximation, the eigenmodes of optical resonators are well known 1. Nonparaxial vector modes have also been calculated in 6 but the mirror shapes were determined from the beam wave front properties (see also $7,8,9$ ) of the non-paraxial beams.

In the present article, we concentrate on two dimensional nearly concentric resonators with circular and parabolic mirror shapes. Although the focusing of plane waves with paraboloid mirrors can be found in several references 10,11,12 (see also 13,14 and references therein), the modes of nearly concentric resonators made of parabolic or circular mirrors have been explicitly determined, to the author's knowledge, solely in 15. The difficulty of such calculations is that the non-paraxial corrections to the propagation equation must be taken into account for fixed mirror shapes.

In 15, the problem was solved using a scalar approximation on the cavity boundaries, as defined in 16. No cross polarization effect, which can appear at the boundaries 17, was taken into account. The resonant modes were determined numerically from a formal non-paraxial mode expansion 18 .

A finding of Ref. 15 is that the beam waist of the non-paraxial fundamental mode of a near concentric cavity made of two parabolic mirrors cannot reach small values as it is the case with the paraxial approximation. Numerical examples were also provided in this reference showing that, for a $1 \mu \mathrm{m}$ radiation wavelength and a mirror radius of curvature of $25 \mathrm{~cm}$, this non-paraxial effect is even sizable down to values of the ratio of the wavelength to the minimum beam size of the order of $\lambda / w_{0} \approx 1 / 30$. This value corresponds to a beam divergence of $\lambda /\left(\pi w_{0}\right) \approx 10 \mathrm{mrad}$ for which noticeable non-paraxial effects are unexpected 19,20. Moreover, these effects cannot be attributed to small Fresnel numbers 21,22 since the mirror apertures are infinite in the calculations of 15 .

Since a nearly concentric cavity made of parabolic mirrors is envisaged for one of the high energy physics experiment mentioned above, it appears necessary to confirm this result. This is the main purpose of the present article. The other purpose of the present work is to setup a numerical method capable to describe easily the effect of the thermoelastic mirror deformation 23 on the non-paraxial modes and more complex geometries as non-planar four mirrors cavities 24 .

Unlike in 15 , the 'brutal force' is employed here to calculate numerically the resonant modes of a 'not so concentric' 2D resonator. A scalar field diffraction theory will be used together with some approximations for the boundary conditions. One of these approximations is the one used in 15, namely the scalar approximation on the boundary 16 , the other one is more accurate in the sense that the curved mirror boundaries are taken into account in the diffraction integral as in the original work of 25. The straightforward numerical methods described long time ago in 26 are used. More refined numerical techniques $27,28,29$, which should first be adapted to the non-paraxial wave propagation or curved boundaries, are not considered here.

The main result of this article is that the calculation of the resonant modes of a nearly concentric cavity is very sensitive to the assumption made to describe the boundary conditions. Assuming the 
same boundary description as in 15 we observe numerical instabilities which increase as the cavity becomes more and more concentric. These numerical instabilities are cured when the mirror shapes are taken into account in the curvilinear diffraction integral.

This article is organized as follows. The integral diffraction formalism is introduced in section II. The numerical techniques are described in section III and some numerical results are given in section IV.

\section{FORMALISM}

A monochromatic continuous electromagnetic wave propagating inside a two dimensional cavity made of two identical curved mirrors $\mathrm{M}_{1} \mathrm{M}_{2}$ is considered (see Figure 1). As in 15, the resonant modes of cavities made of two parabolic and two circular mirrors will be compared. The notations and formula of 16 will be used.

The first Rayleigh-Sommerfeld diffraction integral is used to relate the field $\phi_{\Gamma 1}$ on M1 to the field $\phi_{2}$ incident on $\mathrm{M}_{2}$ :

$$
\phi_{2}\left(\mathbf{r}_{2}\right)=-\int_{\Gamma_{1}} \phi_{\Gamma_{1}}\left(\mathbf{r}_{1}\right) K\left(\mathbf{r}_{1}, \mathbf{r}_{2}\right) d l
$$

where $K\left(\mathbf{r}_{1}, \mathbf{r}_{2}\right)$ is the normal derivative of the free space 2D Green's function and where the vectors $\mathbf{r}_{1}=\left(x_{1}, z_{1}\right)^{t}$ and $\mathbf{r}_{2}=\left(x_{2}, z_{2}\right)^{t}$ are defined in Fig. 1. The integration domain $\Gamma_{1}$ is defined on $\mathbf{M}_{1}$ and, together with the definition of the field $\phi_{\Gamma 1}$, depends on the approximation used to solve the problem 16. In this article two simple cases will be considered: the scalar approximation on the boundaries 16 and a direct integration of (1) on the curved boundaries 25. An accurate 2D solution of the problem would involve a Boundary Element Method (BEM), as described in 16. Since the cavity mirror coatings are made of multilayer stacks 30 deposited on a curved substrate, the BEM solution would be quite mathematically involved and will not be considered here. Moreover, we shall neglect the diffusion and absorption induced by the multilayer coatings of the cavity mirrors.

According to the two boundary approximations described above, (1) may be written as follows

$$
\phi_{2}\left(\mathbf{r}_{2}\right)=-\int_{x_{\min }}^{x_{\max }} \rho_{1} \phi_{1}\left(\mathbf{r}_{1}\right) K\left(\mathbf{r}_{1}, \mathbf{r}_{2}\right) F\left(x_{1}\right) d x_{1}
$$

Where $\phi_{\Gamma 1}$ is approximated by the reflected field $\rho_{1} \phi_{1}$ on $\mathrm{M}_{1}$ (with $\rho_{1}$ the reflection coefficient which is assumed to be a constant over the mirror surfaces) and where the mirror shapes and the boundary approximations are embodied in the function $F$.

Two expressions of the kernel $K$ will be considered:

- Exact, or non-paraxial 16:

$$
K_{N P}\left(\mathbf{r}_{1}, \mathbf{r}_{2}\right)=\frac{-i k}{4} \frac{\mathbf{n}_{1} \cdot\left(\mathbf{r}_{2}-\mathbf{r}_{1}\right)}{\left|\mathbf{r}_{2}-\mathbf{r}_{1}\right|} H_{1}^{(2)}\left(k\left|\mathbf{r}_{1}-\mathbf{r}_{2}\right|\right)
$$

where $H_{1}^{(2)}$ is the first order Hankel function of second kind and where $\mathbf{n}_{\mathbf{1}}$ is the normal unit vector on $\mathrm{M}_{1}$.

- Paraxial approximation

$$
K_{\text {Parax }}\left(\mathbf{r}_{1}, \mathbf{r}_{2}\right)=\frac{k}{4} \sqrt{\frac{2}{k \pi L}} \exp \left(i \frac{\pi}{4}\right) \exp \left(-i k L\left(1+\frac{x_{1}^{2}}{2 L^{2}}-\frac{x_{1} x_{2}}{L^{2}}\right)\right)
$$




\section{Scalar approximation on the boundaries}

This approximation, henceforth referred as approximation $\mathrm{A}$, is used in the calculations of 15 . In this case, the function $F$ reads $F\left(x_{1}\right)=\exp \left(2 i k \Delta\left(x_{1}\right)\right)$ such:

- $\Delta\left(x_{1}\right)=x_{1}^{2} /(2 R)$ for parabolic mirrors;

- $\Delta\left(x_{1}\right)=R-\sqrt{R^{2}-x_{1}^{2}}$ for circular mirrors.

In these expressions, $R$ is the mirror radius of curvature. Since this approximation assumes flat boundaries, the inclination factor of kernel (3) is simply given by $\mathbf{n}_{\mathbf{1}} \cdot\left(\mathbf{r}_{2}-\mathbf{r}_{1}\right)=L$, where $L$ is the distance between the two mirrors (see Figure 1).

Note that this approximation is derived for a plane wave which may not be that adequate in the case of a strongly focused beam.

\section{Curvilinear integral on the boundaries}

Here, the mirror shapes are taken into account in the integral of (1). This is still an approximation with respect to the accurate BEM method, though more accurate than approximation A. This approximation will be hereafter referred as approximation B. For circular mirrors, the function $F$ and the geometric vectors needed to compute (2) and (3) are given by:

$$
F\left(x_{1}\right)=\frac{R}{\sqrt{R^{2}-x_{1}^{2}}}, \mathbf{n}_{1}=\frac{-1}{R}\left(\begin{array}{c}
x_{1} \\
\sqrt{R^{2}-x_{1}^{2}}
\end{array}\right), \mathbf{r}_{1}=\left(\begin{array}{c}
x_{1} \\
\sqrt{R^{2}-x_{1}^{2}}-\varepsilon
\end{array}\right)
$$

where similar expressions hold for $\mathbf{r}_{2}$ and where $\varepsilon=R-L / 2$. In order to study the influence of the mirror shape on the cavity fundamental mode, we also introduce a curved mirror of equation

$$
z_{1}=R-\frac{x_{1}^{2}}{2 R}-\frac{x_{1}^{4}}{\varsigma R^{3}}-\varepsilon
$$

corresponding to a parabolic mirror when $\varsigma \rightarrow \infty$ and approximately to a circular mirror when $\zeta=8$. This expression was indeed used to describe the mirror shapes in 15 . The related function $F$ and geometric vectors read:

$$
F\left(x_{1}\right)=\sqrt{1+\frac{x_{1}^{2}}{R^{2}}\left(1+4 \frac{x_{1}^{2}}{\varsigma R^{2}}\right)^{2}}, \mathbf{n}_{1}=\frac{-1}{\sqrt{\frac{\varsigma^{2} R^{6}}{\left(\varsigma R^{2}+4 x_{1}^{2}\right)^{2}}+x_{1}^{2}}}\left(\begin{array}{c}
x_{1} \\
\frac{\varsigma R^{3}}{\varsigma R^{2}+4 x_{1}^{2}}
\end{array}\right), \mathbf{r}_{1}=\left(\begin{array}{c}
x_{1} \\
R-\frac{x_{1}^{2}}{2 R}-\frac{x_{1}^{4}}{\varsigma R^{3}}-\varepsilon
\end{array}\right) .
$$

Note that, by defining the local curvature of the mirror as $\left\|\mathrm{dt}_{1}(l) / \mathrm{d} l\right\|^{-1}$, where $\mathbf{t}_{\mathbf{1}}$ is the tangent vector perpendicular to $\mathbf{n}_{\mathbf{1}}$, one sees that the higher $\zeta$, the higher the average curvature of the mirror.

\section{Determination of the resonant modes}

Let us first determine the resonant modes using the round trip RT1 depicted in Figure 1. The relation between the fields on mirrors $\mathrm{M}_{1}$ and $\mathrm{M}_{2}$ is given by Eq. (2) and the resonant modes are defined, for 
both approximations $\mathrm{A}$ and $\mathrm{B}$, by the condition

$$
\gamma \phi_{1}\left(x_{1}\right)=\phi_{2}\left(x_{2}\right) \forall x_{1} \mid x_{2}=x_{1}
$$

where $\gamma$ is the cavity mode loss and where this relation holds because the mirrors are assumed to be identical. These modes will be denoted by $\phi^{(n)}(x, z)$.

Once an eigenmode has been found, the 2D electric field $\mathbf{E}^{(\mathbf{n})}(x, z)=\left(E_{\perp}^{(n)}(x, z), E_{z}^{(n)}(x, z)\right)^{t}$, where the subscript $\perp$ indicates the $x$ or $y$ axis, can be estimated 15,31 for a given value of $z$ :

$$
\begin{aligned}
& E_{\perp}^{(n)}(x, z) \approx \phi^{(n)}(x, z), \\
& E_{z}^{(n)}(x, z) \approx F T^{-1}\left[\frac{i k_{x}}{\sqrt{k^{2}-k_{x}^{2}}} F T\left[\phi^{(n)}(x, z)\right]\right]
\end{aligned}
$$

where FT stands for the Fourier Transformation and with $k=2 \pi / \lambda$. The second equation of (7) comes from the condition $\nabla . E=0$. However, although (7) holds for free field propagation, it can just be an estimate of the resonant electric field of a cavity.

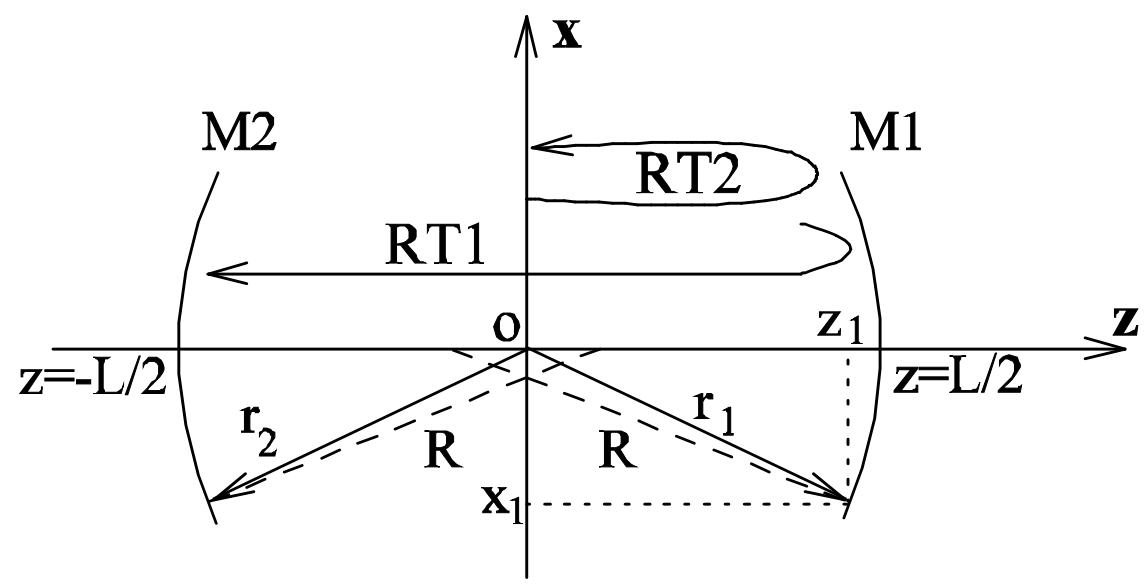

Figure 1 Schematic drawing of a 2D near concentric cavity made of two identical mirrors (here circular). The two round trip considered in the text RTI and RT2 are also depicted.

\section{NUMERICAL METHODS}

When (1) is computed numerically, the $x$ space is sampled $x \rightarrow\left\{x_{j}\right\}_{j=1 \ldots N}$ and the following expression comes out 27:

$$
\gamma \phi\left(x_{m}\right)=\sum_{j}^{N} p_{j} \phi\left(x_{j}\right) F\left(x_{j}\right) K\left(\mathbf{r}_{\mathbf{j}}, \mathbf{r}_{\mathbf{m}}\right)
$$

where $p_{j}$ is a weight which depends on the numerical method under consideration. For a nearly concentric resonator, the $x$ space must be sampled very finely so that typical values for $N$ are $2^{11}$. $2^{12}$. 
For such a high values of $N$, a simple trapeze like method is reliable and the convergence is easily checked by varying the value of $N$. With this method, choosing an integration interval $\left[x_{\min }, x_{\max }\right]$ and a constant spacing $D_{x}=\left(x_{\max }-x_{\min }\right) / N$ between $x$ nodes, one gets $p_{j}=D_{x} \forall j$.

Alternatively, to check our numerical results, the Gauss-Legendre quadrature method was used. The nodes and the weight were determined with the Maple software 32 for a maximum value of $N=300$. It should be mentioned that, for this large sampling value, more than 100 digits were required.

Defining the $N \times 1$ matrix $\Phi=\left(\phi\left(x_{1}\right), \ldots \phi\left(x_{N}\right)\right)^{t}$ and the symmetric non-hermitian $N \times N$ matrix $M$ such $M_{j m}=p_{j} F\left(x_{j}\right) K\left(\mathbf{r}_{\mathbf{j}}, \mathbf{r}_{\mathbf{m}}\right)$, one can write (8) as follows

$$
\gamma \Phi=M \Phi
$$

i.e. the cavity modes are given by the eigenvectors of the matrix $M$. The eigenvalue problem is solved numerically with the Matlab software 33. Note that the code consists in a very few lines and can be implemented very easily.

Hitherto, the round trip RT1 of Figure 1 has been considered. In order to study the accuracy of approximations A and B, the round trip RT2 (see Figure 1) is also used to determine the cavity resonant modes. The modes determined with RT1 and RT2 should be the same although the two reference surfaces are both curved in RT1 whereas one of them is flat in RT2. For this latter roundtrip, two diffraction matrices must be computed, from the plane $z=0$ to $\mathrm{M}_{1}$, forth and back. Denoting by $O$ and $P$ the matrices describing the propagation from $z=0$ to $\mathrm{M}_{1}$ and from $\mathrm{M}_{1}$ to $z=0$ respectively, one gets

$$
\begin{gathered}
O_{j m}=p_{j} K\left(\mathbf{r}_{1 \mathbf{j}}, \mathbf{r}_{\mathbf{2 m}}\right) \mid z_{1 j}=0, \text { and }\left\{\begin{array}{l}
\operatorname{approx} . \mathrm{B}:\left(x_{2 m}, z_{2 m}\right) \in \mathrm{M}_{1} \\
\operatorname{approx} \mathrm{A}: z_{2 m}=L / 2
\end{array}\right. \\
P_{j m}=p_{j} K\left(\mathbf{r}_{1 \mathbf{j}}, \mathbf{r}_{\mathbf{2 m}}\right) F\left(x_{1 j}\right) \mid z_{2 j}=0, \text { and }\left\{\begin{array}{l}
\operatorname{approx} . \mathrm{B}:\left(x_{1 m}, z_{1 m}\right) \in \mathrm{M}_{1} \\
\operatorname{approx} \mathrm{A}: z_{1 m}=L / 2
\end{array}\right.
\end{gathered}
$$

Thus, the matrix $M$ of eq.(9) simply reads $M=O P$ for RT2.

All the numerical calculations were checked by comparing the formal paraxial solutions 1 with the fundamental eigenvector of the matrix $M$ built with the paraxial kernel of (4). A perfect agreement was found for all the results shown in the next section.

As a benchmark for our numerical results, we are able to reproduce the figures 4 and 5 of 15 using the diffraction integral (1) with the kernels (3) or (4) and the field definition (7). Figure 2 shows the intensity profile of Gaussian beam of waist $w_{0}=1 \mu \mathrm{m}$ propagated over ten Rayleigh lengths (the wavelength was fixed to $\lambda=1064 \mathrm{~nm}$ ). A numerical agreement was also found with a calculation using the angular spectrum propagation 34 and therefore with the free space propagation method of 15 . 


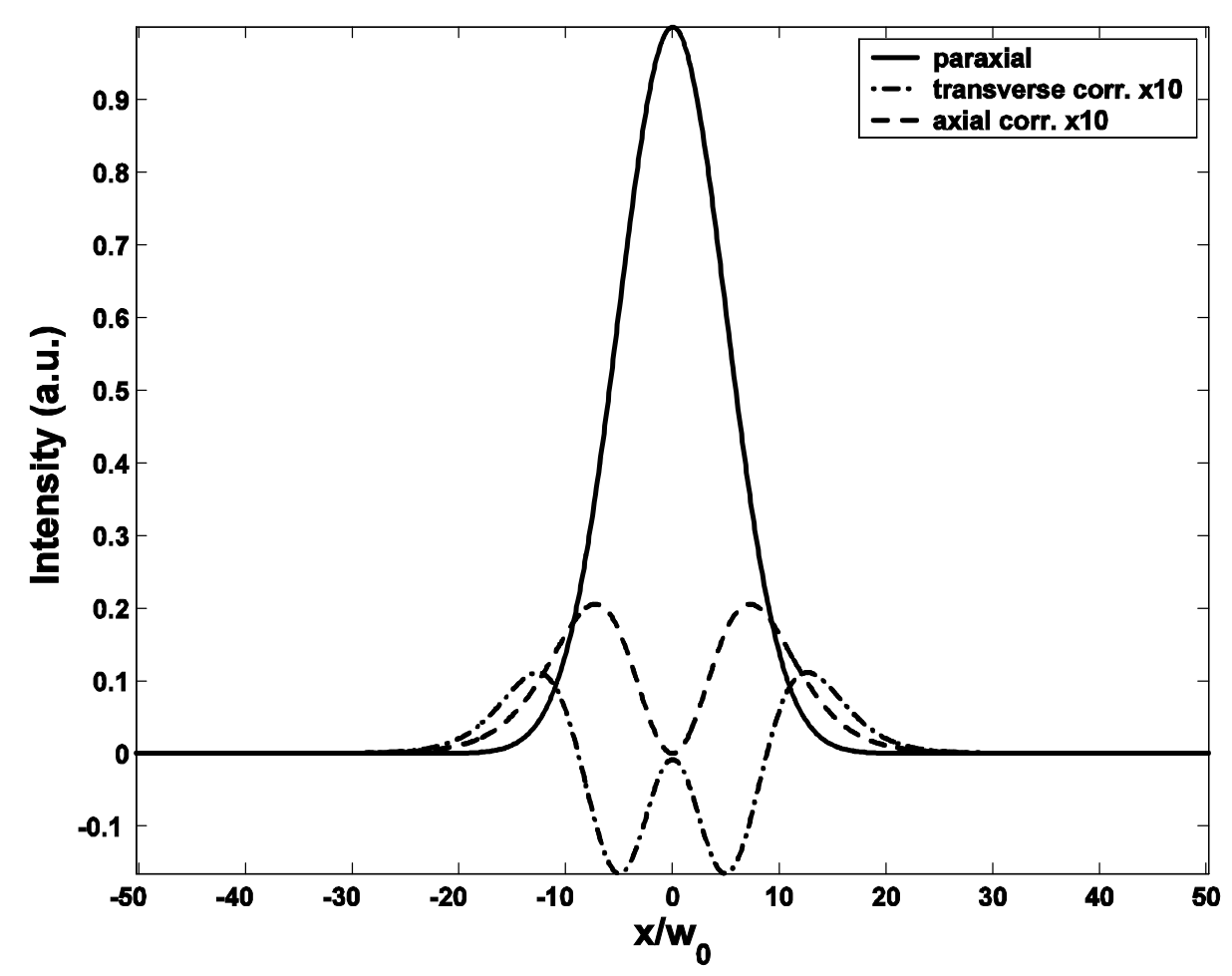

Figure 2 Intensity profile at $29.5 \mu \mathrm{m}$ from the position of the minimum waist $w_{0}=1 \mu \mathrm{m}$ of a Gaussian beam. Full line: paraxial propagation. Dashed line: longitudinal correction $E_{z}$. Dashed-dotted line: difference between the non-paraxial and paraxial scalar propagations.

\section{NUMERICAL RESULTS}

As in 15 , the mirror radius of curvatures are fixed to $R=0.25 \mathrm{~m}$ and the wavelength to $\lambda=1064 \mathrm{~nm}$. The integration boundary $x_{\max }$ (with $x_{\min }=-x_{\max }$ ) is changed according to the approximation used and to the degree of concentricity. Denoting by $w_{0}$ and $w_{\mathrm{M}}$ the paraxial waist at $z=0$ and $z=L / 2$ respectively, we fix $x_{\max }=6 w_{M}$ and $x_{\max }=8 w_{M}$ for the approximations B and A respectively.

However, as indicated in the introduction, the aim of the present work is to describe cavity modes having transverse sizes much smaller that the mirror apertures. One must then verify that diffraction losses introduced by the final integration range, which acts in fact as a numerical aperture, are negligible. These losses can be estimated from the Fresnel number $N_{F}=x_{\max }^{2} / \lambda L 25$ shown in Figure 3(a) as a function of the cavity concentricity. Although the above expression of $N_{F}$ is related to the Fresnel approximation of the diffraction kernel (3) 35 and that a non-paraxial expression should be derived 36, Figure 3(a) shows that diffraction losses related to the finite integration interval are negligible 25,37 .

The finite integration range also acts as a cutoff on the transverse mode orders. This cutoff can be estimated by the ratio $N_{\max }=w_{\mathrm{M}} / x_{\max }, 38$ which gives here $N_{\mathrm{ma}}=36,64$ for the values of $x_{\max }$ given above.

The effect of the finite integration range on the shape of the cavity modes was further checked to be negligible when varying $x_{\max }$ from $5 w_{\mathrm{M}}$ to $12 w_{\mathrm{M}}$. It was also found that, in order to get the physical modes among the large number of 'noisy' eigenvectors of the matrix $M$, the node spacing needs to be smaller than $w_{0}$. This led us with a number of nodes $N=2^{12}$ and $2^{11}$ for the approximations A and B respectively. 
The longitudinal contributions (see (7)) to the intensity distributions presented here are smaller than the relative level of $10^{-6}$, they are therefore neglected. The difference between the calculations performed with the circular shape (5) and its fourth order expansion (6) with $\zeta=8$ ) was found to be negligible. One can thus directly compare our numerical results for circular mirror shapes with those of 15 where the fourth order expansion was used.

(a)

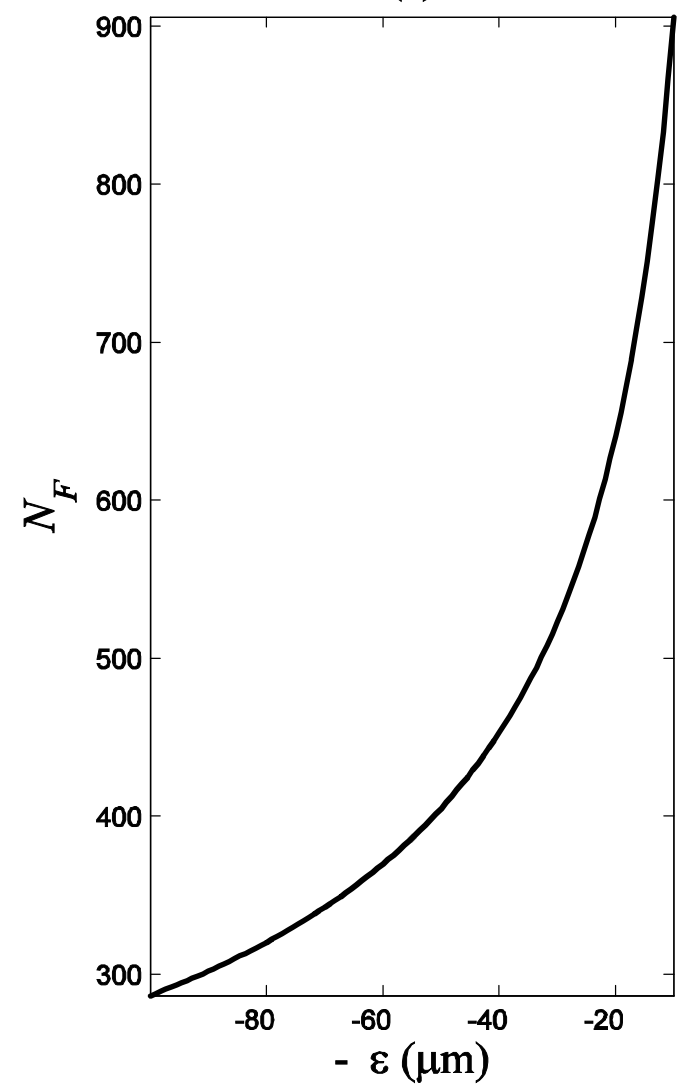

(b)

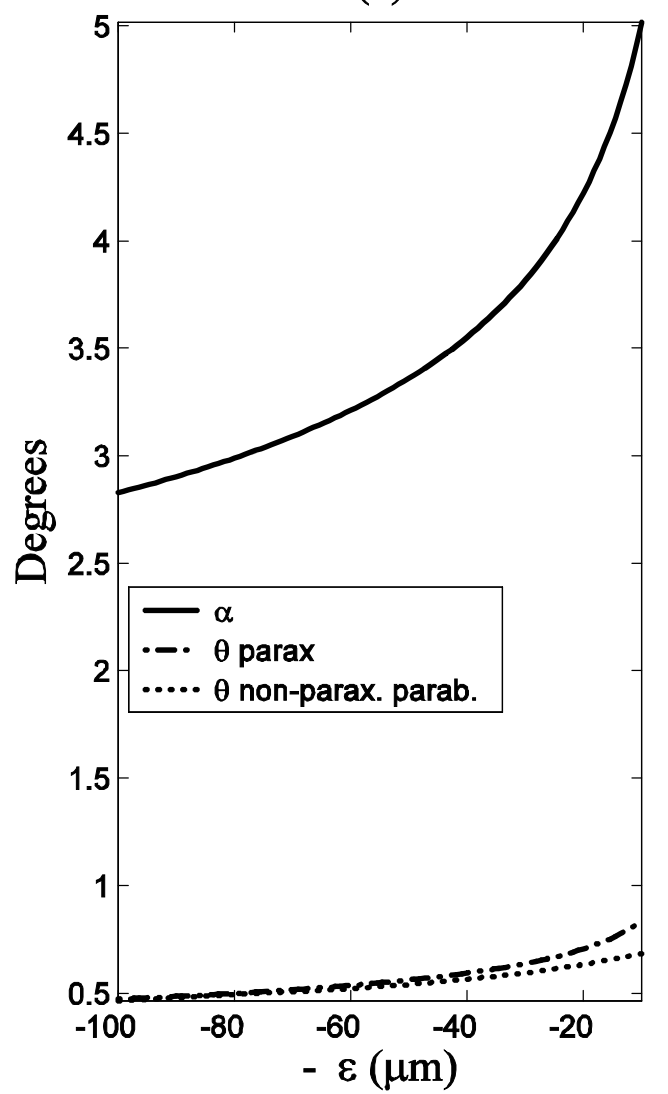

Figure 3 (a) Fresnel number as a function of $\varepsilon$. (b) Angular aperture $\alpha$ and beam angular divergence $\theta$ as a function of $\varepsilon$. In figure (b), the paraxial fundamental mode for circular mirrors (dashed line) and the nonparaxial fundamental mode computed with approximation $B$ for parabolic mirrors (dashed-dotted line) have been considered.

Examples of intensity profiles corresponding to the fundamental modes are plotted in Figure 4. Very large differences between approximations $\mathrm{A}$ and $\mathrm{B}$ are observed for a rather large concentricity $\varepsilon=30 \mu \mathrm{m}$. Looking at the tails $|x| / w_{0}>>1$ of the solutions obtained with approximation $\mathrm{A}$, one can see that, extrapolating to infinity, the intensity profile may not be an integrable function. This means that the solutions obtained with approximations A are most likely non-physical and thereby that this approximation should be used carefully for concentric resonators.

The beam widths at the cavity centre and on the mirror surfaces are shown in Figure 5 and Figure 6 respectively. As in 15, the widths are computed from the second moment of the intensity distributions. These figures show that, as the cavity becomes more and more concentric, the approximation A becomes inaccurate and the modes computed with two different round trips differ. With approximations B, one can see that non-paraxial corrections do not generate aberrations when circular mirrors are used. However, with parabolic mirrors, a deviation from the paraxial solution is observed even with approximation B. Within this approximation, the modes computed with the round trips RT1 and RT2 coincide. 


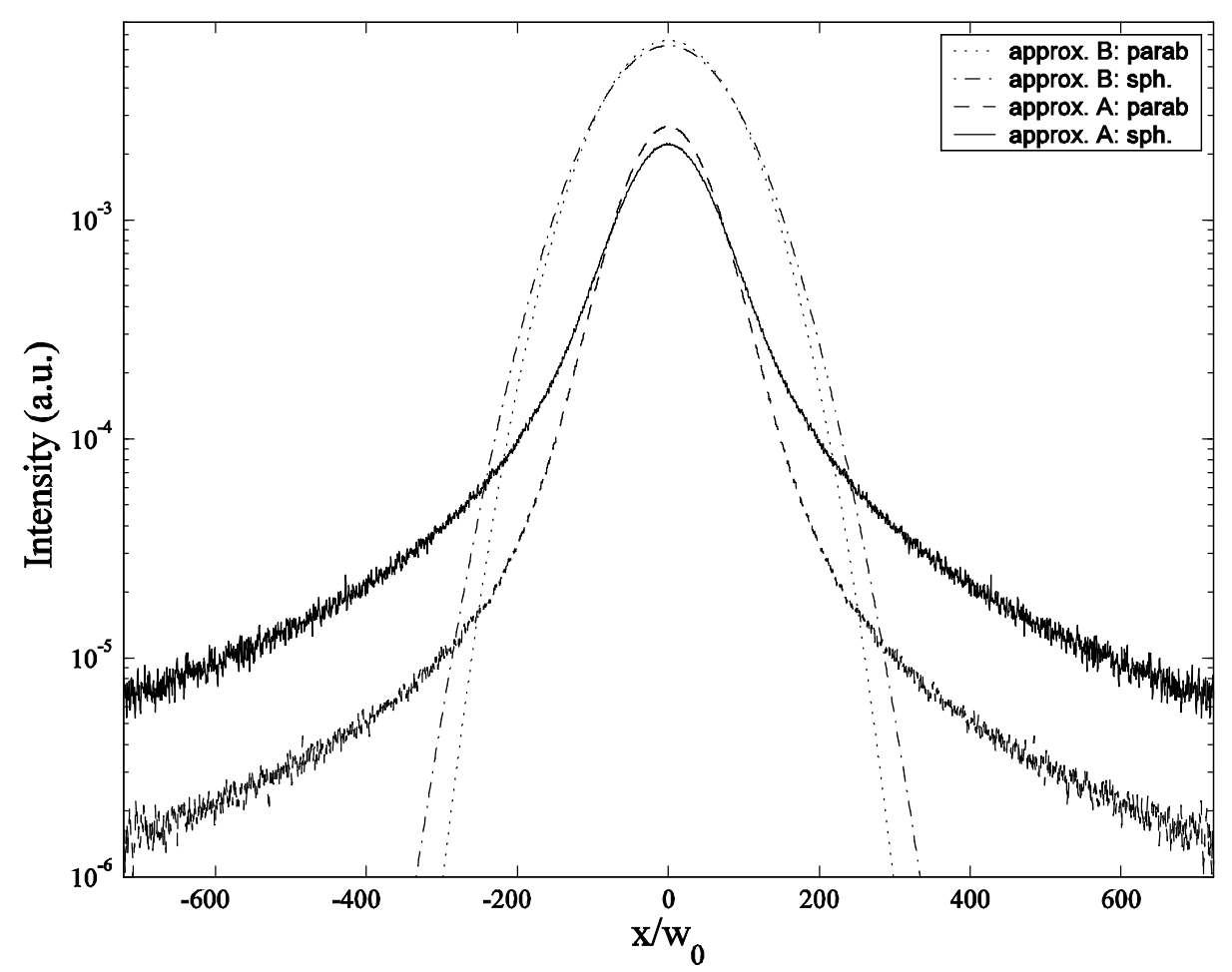

Figure 4 Intensity profile on the mirror surface of the fundamental modes. Calculations are performed according to the approximations $A$ and $B$, for the round-trip RT1 and for two mirror shapes (parabolic and circular). The concentricity parameter is fixed to $\varepsilon=30 \mu \mathrm{m}$.

The fact that non-paraxial corrections are noticeable for parabolic mirror was already reported in 15 . But an intriguing fact is that approximation $\mathrm{A}$ is used in this reference. At first sight, the curves of

Figure 5 and Figure 6 related to the approximation B appear similar to those of Figures 6 and 7 of 15. However, this is most likely due to a numerical hazard. Figure 7 shows the intensity profiles of the excited mode corresponding to Figure 8 of 15. A large difference is noticeable between these two figures, thus indicating that the numerical results of the present article differ from those of 15 for small concentricity values.

As already mentioned, the consistency of the numerical results presented here, which depend on the values of $x_{\max }$ and $N$ actually, are checked by comparing the paraxial modes obtained numerically with the kernel of (4) and the approximation A, and the formal expression of 1 . A perfect agreement was observed for all the values of $\varepsilon$ considered here. The results are also stable against variations of $x_{\max }$ and $N$. Moreover, as indicated in section III, the Gauss-Legendre numerical method was also used to solve (8) and (9). Since the number of nodes is limited to $N=300$, the numerical results were checked on a limited range of integration $\left(x_{\max }=2 w_{M}\right.$ for the approximation A). An excellent agreement was observed between the two methods of section II.

Since the numerical method utilized here was checked to be accurate enough, we have identified three possible explanations for the disagreement between our results and those of 15. Firstly, according to the mean value theorem for integrals, there is no mathematical approximation in the writing of eqs. (8)-(9) provided $\phi, F$ and $K$ are continuous functions. The only mathematical approximation rests in the choice of the $x$ nodes and is obviously controlled by reducing the nod spacing $D_{x}$. The eigenvectors determined here thus belong to a large class of functions since the only constraint is the continuity in the range $\left[-x_{\max }, x_{\max }\right]$. This is different with the method used in 15 where the cavity mode is expended as a series of the non-paraxial free space modes. These modes are continuous functions which belong to the space of square integrable functions and therefore to a subspace of the solution space of (8) and (9). Secondly, as pointed out in 39, where a 
method similar to the one of 15 is used, the truncation of the modal series is strongly correlated to the choice of the dimensioned parameter appearing in the argument of the Hermite-Gauss functions. Since very little numerical information are given in 15 , it may also be that the dimension of the functional space of the solution has been reduced by the numerical choices. Thirdly, the convergence of the modal series of 15 may not be fast enough in some particular cases so that the series truncation could lead to numerical instabilities.

The angular aperture $\alpha=\tan ^{-1}\left(x_{\max } / R\right)$ and the beam divergence $\theta=\lambda /\left(\pi w_{0}\right)$ are shown in Figure 3 (b) as a function of $\varepsilon$. For the range of concentricity considered here, one has $\alpha<5^{\circ}$ and $\theta<1^{\circ}$ for both circular and parabolic mirrors. Since $N_{F}>300$ (see Figure 3(a)), it is astonishing that the nonparaxial effects come out to be so large for parabolic mirrors. To verify that these effects are related to the mirror shapes, we have computed the fundamental eigenmodes for various values of the parameter $\zeta$ of eq. (6),0. The beam widths at the cavity centre and on the mirrors are shown in Figure 8 for $\varepsilon=30 \mu \mathrm{m}$ as a function of $\zeta$. This figure shows that the smaller the average curvature of the mirror, the smaller the beam waist and that a non-paraxial effect of the order of $10 \%$ are observed for a small variation of $\zeta$ from 5 to 10 .

One way to get an insight into our results would be to compare with non-paraxial scalar beams 40,41. However, in the present work the two dimensional Green's function is used to construct the diffraction kernel (3) and, to be able to compare with 40,41 one should have used the three dimensional Green's function integrated over the azimuth angle. Since the later is not reducible to the former we postpone this comparison to a future publication where we shall solve the problem in three dimensions.

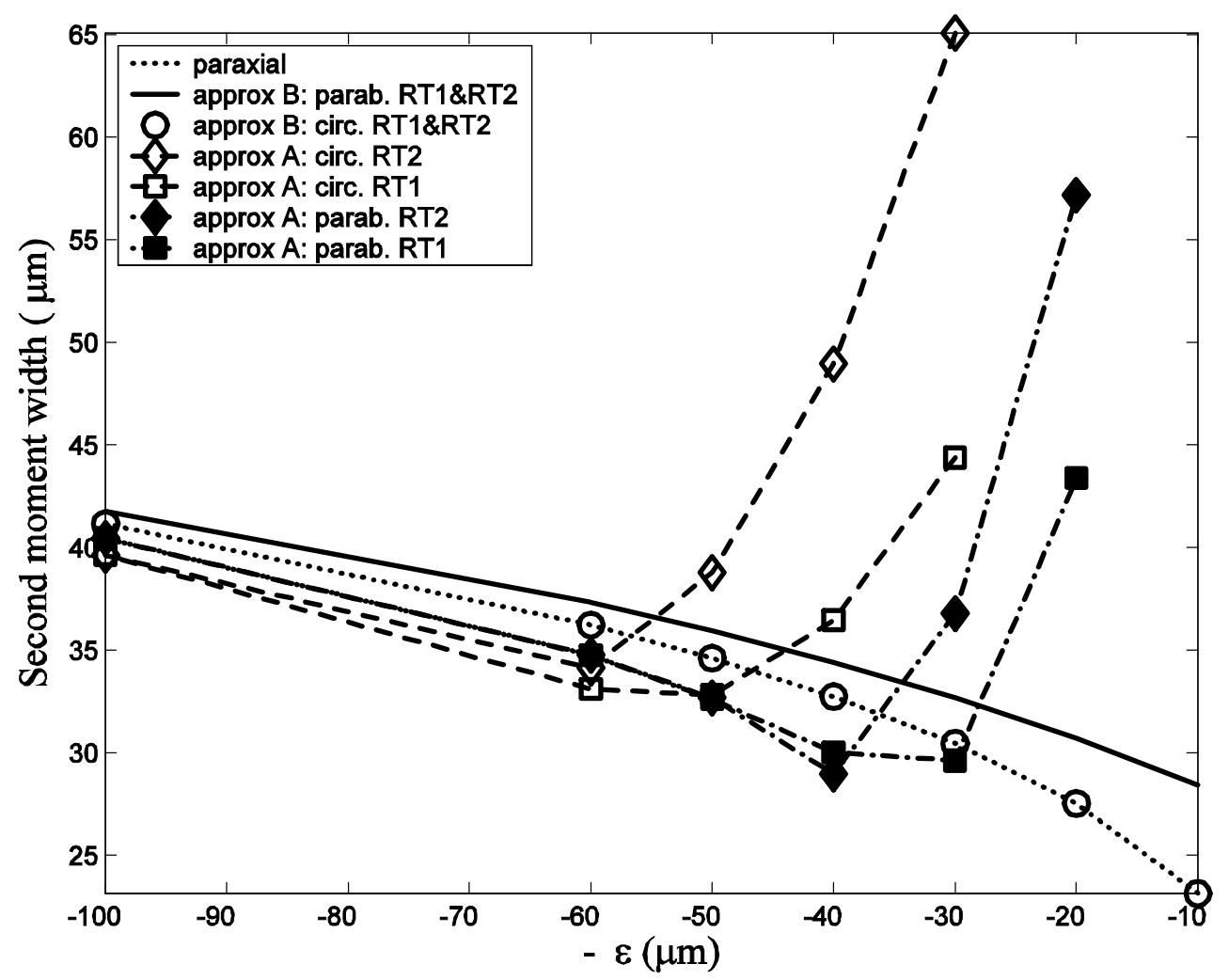

Figure 5 Width of the fundamental mode at the cavity centre $(z=0)$ as a function of the concentricity parameter $\varepsilon$ Calculations performed with the approximations $A$ and $B$ and for the two round trips RTI and RT2 are compared. Note that the results obtained with approximation $B$ for circular mirrors (open circles) coincide with the paraxial case (dotted curve.) 


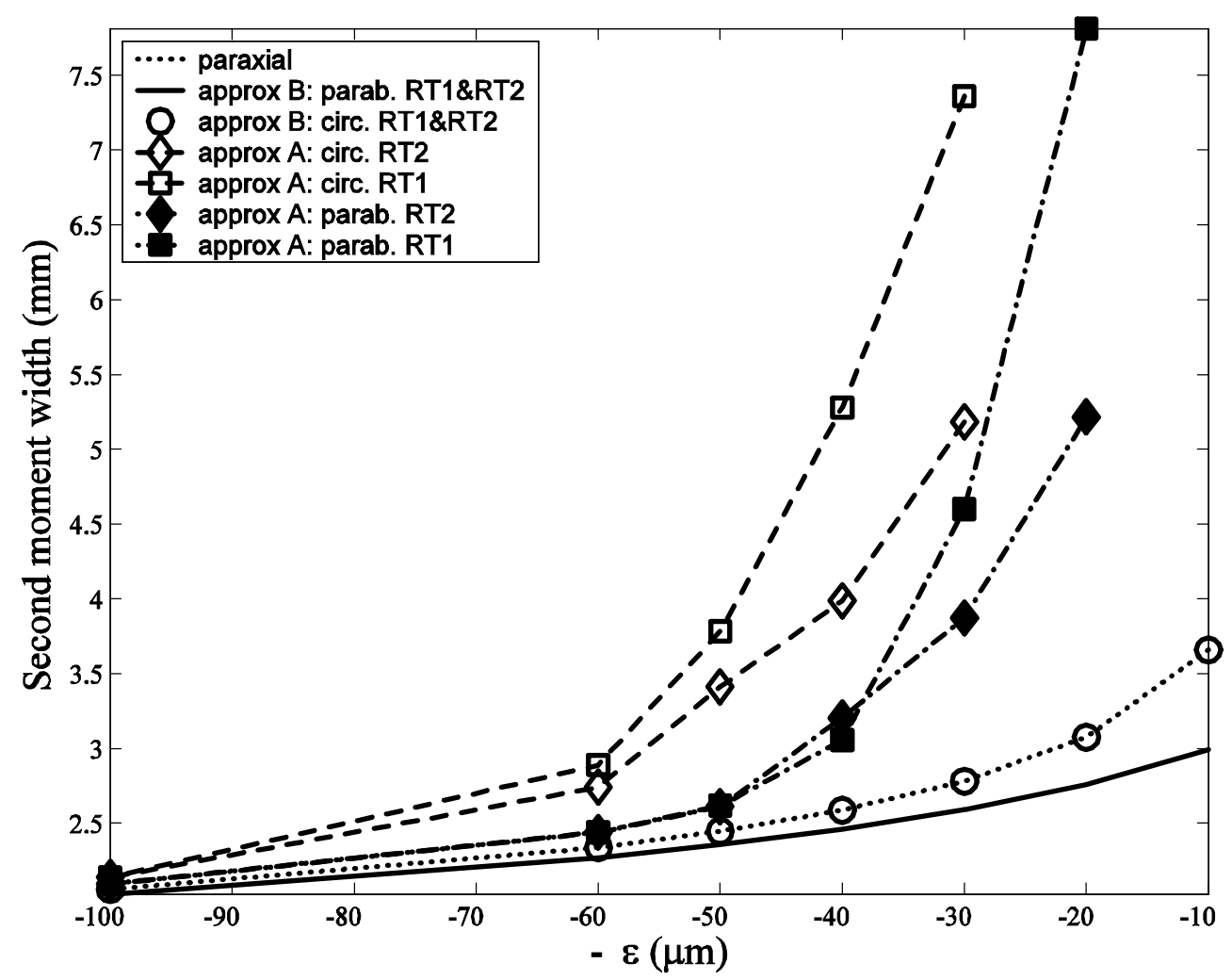

Figure 6 Width of the fundamental mode on the cavity mirrors as a function of the concentricity parameter $\varepsilon$. Calculations performed with the approximations $A$ and $B$ and for the two round trips RT1 and RT2 are compared. Note that the results obtained with approximation $B$ for circular mirrors (open circles) coincide with the paraxial case (dotted curve.)

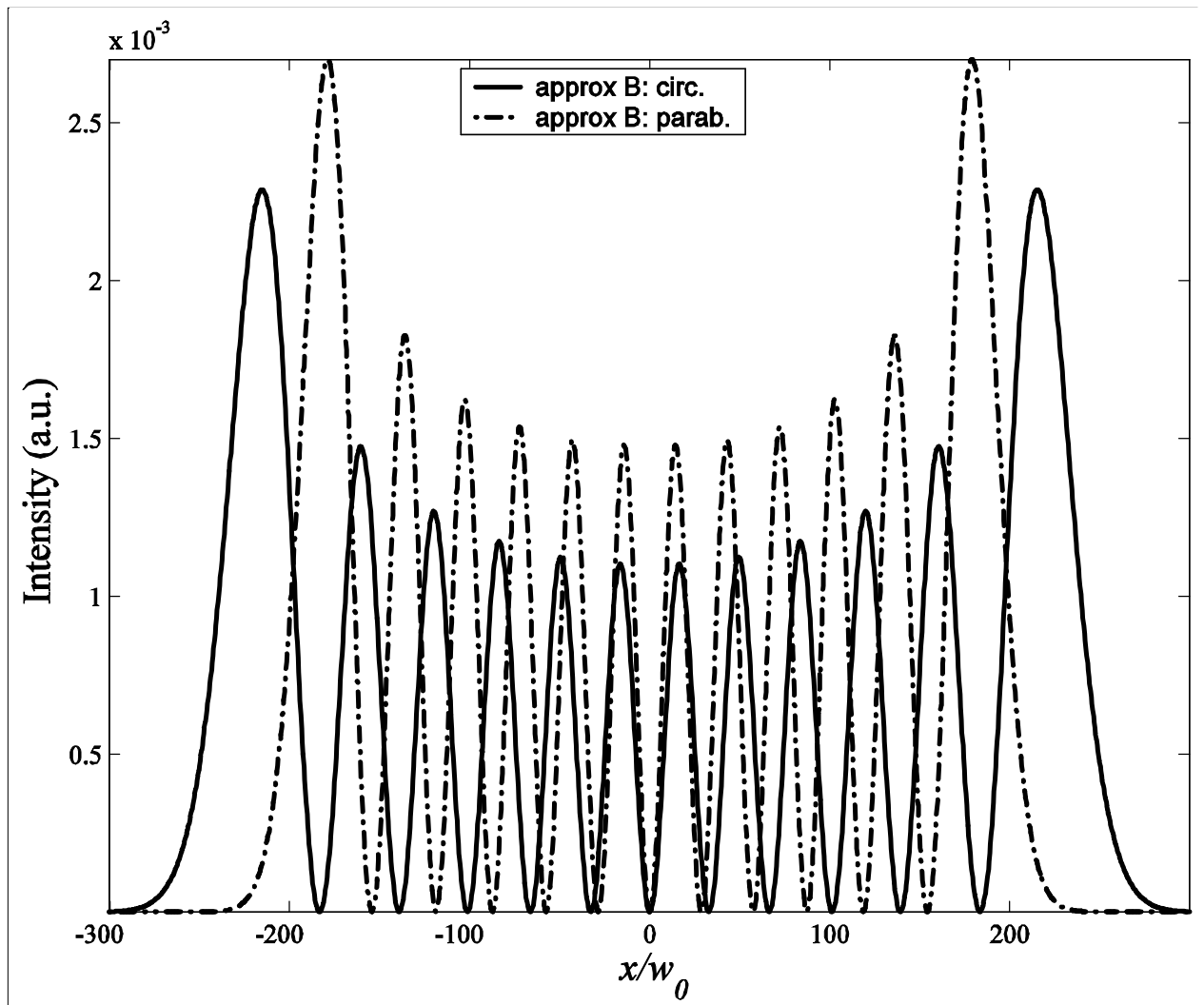

Figure 7 Intensity profile on the mirror surface of an exited mode. The calculations are performed using the approximation $B$ for the two mirror shapes, parabolic and circular. The concentricity parameter is fixed to $\varepsilon=50 \mu \mathrm{m}$. 


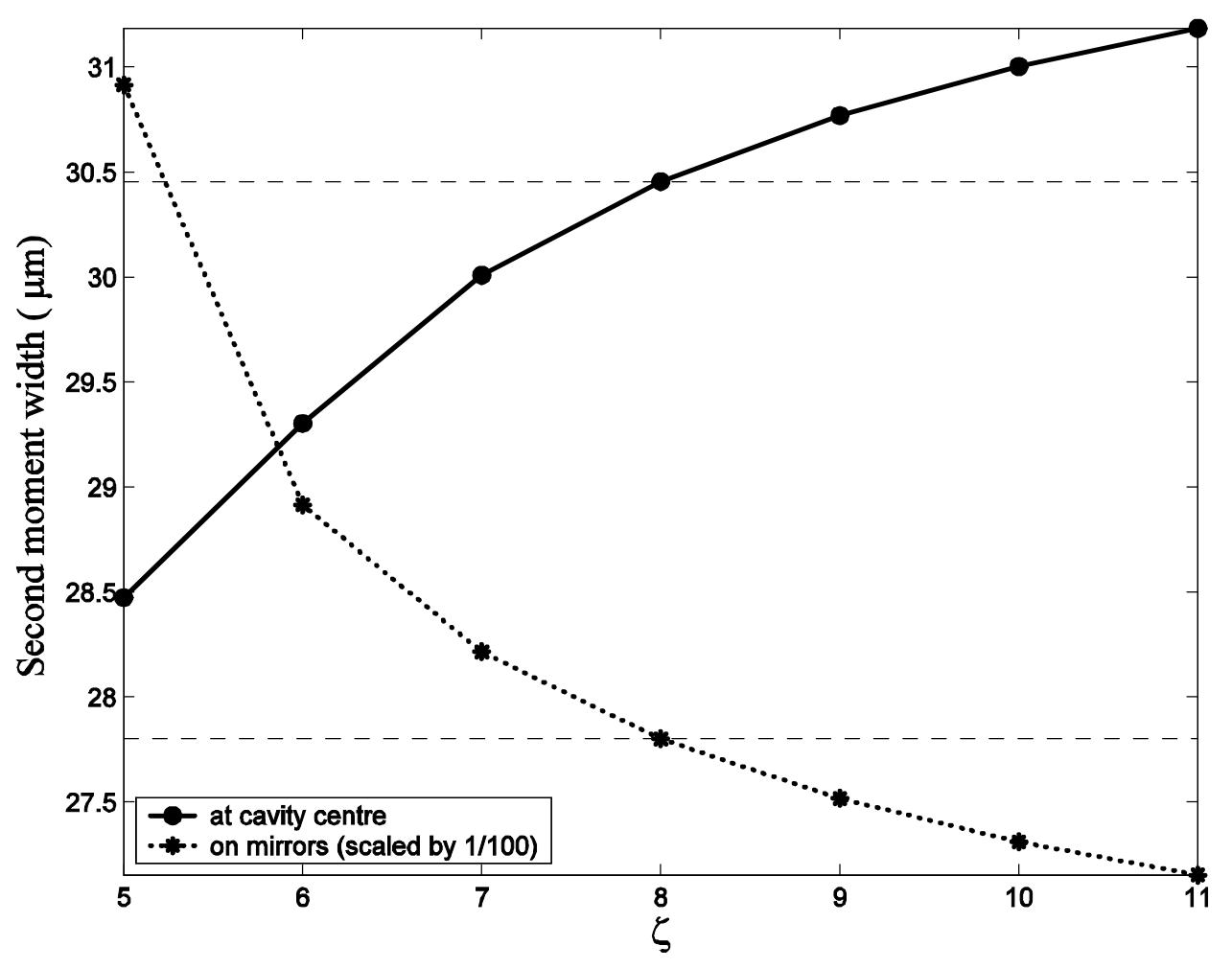

Figure 8 Second moment width of the fundamental mode on the cavity mirrors (full line) and at the cavity centre (dotted line, scaled by 1/100) as a function of the mirror shape parameter $\zeta$ Approximation $B$ is used for $\varepsilon=30 \mu \mathrm{m}$. The two horizontal dashed lines represent the results of the paraxial approximation.

\section{CONCLUSION}

The aim of the work presented here was to confirm the finding of 15 using a simple and straightforward numerical method. We have thus calculated the resonant modes of a 2D nearly concentric cavity with two approximations for the boundary conditions. When the approximation of 15 is used for a cavity becoming concentric, discrepancies are observed between our numerical results and those of 15 . Several numerical cross-checks of our numerical procedure were performed, validating thus the numerical accuracy of the results described in section IV. We then pointed out that the reason for the observed discrepancies relies most likely on the mathematical assumptions related to the numerical method used to solve the eigenvalue problem, and therefore that the scalar approximation on the boundary is not reliable for a concentric resonator.

As reported in 15 from the numerical example also employed in the present article, we also observed that aberrations appear already for $\lambda / \mathrm{w}_{0} \approx 1 / 40$ when the cavity is made of parabolic mirrors. This is an intriguing fact since the Fresnel numbers considered here are very large. Varying the mirror shapes, we observed that for a given concentricity the higher the average curvature of the mirror the higher the beam waist at the cavity centre.

In order to confirm this result, one should first solve the problem using the BEM and next to compute the vector modes which could then have sizable effects 42 . These will be the subjects of a future publication. 


\section{ACKNOWLEDGMENT}

We would like to thank the Reviewers for their fruitful suggestions which have improved this publication.

\section{REFERENCES}

1. H. Kogelnick and T. Li, "Laser beams and resonators", Appl. Opt., vol. 5, pp. 1550-1567, 1966.

2. R.J. Loewen, "A compact light source: design and technical feasibility study of a laser-electron storage ring X-ray source,” Ph.D. dissertation, Dept. Phys., Stanford Univ., Stanford, CA, 2003.

3. G. Moortgat-Pick et al., "The Role of polarized positrons and electrons in revealing fundamental interactions at the linear collider", e-print archive hep-ph/0507011, submitted to Phys. Rep. Available: http://arxiv.org/PS cache/hep-ph/pdf/0507/0507011.pdf.

4. S. Araki et al., "Conceptual design of a polarized positron source based on laser Compton scattering", e-Print archive physics/0509016. Available: http://arxiv.org/PS_cache/physics/pdf/0509/0509016.pdf.

5. A.N. Luiten and J.C. Petersen,"Ultrafast resonant polarization interferometry: towards the first direct detection of vacuum polarization", Phys. Rev. A, vol. 70, pp. 033801-033809, 2004.

6. A.L. Cullen and P.K. Yu, "Complex source-point theory of the electromagnetic open resonator", Proc. R. Soc. Lond. A, vol. 366, pp. 155-171, 1979.

7. L.W. Davis, "Vector electromagnetic modes of an optical resonator", Phys. Rev. A, vol. 30, pp3092-3096, 1984.

8. P. Varga and P. Török, "Exact and approximate solutions of Maxwell's equations for a confocal cavity", Opt. Lett., vol 21, pp 1523-1525,1996.

9. S.R. Seshadri, "Electromagnetic theory of an open resonator", J. Opt. Soc. Am. A, vol 18, pp 1748-1757, 2001.

10. C.J.R. Sheppard, A. Choudhury and J. Gannaway, "Electromagnetic field near the focus of wide-angular lens and mirror systems", Micro. Opt. and Acoust., vol. 1, pp. 129-132, 1977.

11. R. Barakat, "Diffracted electromagnetic fields in the neighborhood of the focus of a paraboloidal mirror having a central opturation", Appl. Opt., vol. 26, pp. 3790-3795, 1987.

12. P. Varga and P. Török, "Focusing of electromagnetic waves by paraboloid mirrors. I. Theory", Opt. Soc. Am. A, vol. 17, pp2081-2089, 2000.

13. J.J. Stamnes, Waves in focal regions: propagation, diffraction, and focusing of light, sound, and water waves, A. Hilger, Bristol 1986, p. 455.

14. M. Kline and I.W. Kay, Electromagnetic theory and geometrical optics, J. Wiley, New-York 1965 , p. 350.

15. H. Laabs and A.T. Friberg, "Nonparaxial eigenmodes of stable resonators", IEEE J. Quant. El., vol. 35, pp. 198-207, February 1999.

16. .J.M. Bendickson, E.N. Glytsis and T.K. Gaylord, "Scalar integral diffraction methods: unification, accuracy, and comparison with rigorous boundary element method with application to diffractive cylindrical lenses", J. Opt. Soc. Am. A vol. 15, pp. 1822-1837, 1998.

17. P.M. Morse and H. Feshbach, Methods of theoretical physics, Mc Graw-Hill, New-York, 1953. See Chap. 5.

18. H. Laabs, "Propagation of Hermite-Gaussian-beams beyond the paraxial approximation", Opt. Comm., vol. 147, pp. 1-4, 1998. 
19. M. Lax, W.H. Louisell and W.B. McKnight, "From Maxwell to paraxial wave optics", Phys. Rev. A, vol. 11, pp. 1365-1370, 1975.

20. P. Varga and P. Török, "The Gaussian wave solution of Maxwell's equations and the validity of scalar wave approximation", Opt. Comm., vol. 152, pp. 108-118, 1998.

21. Y. Li and E. Wolf, "Focal shifts in diffracted converging spherical waves", Opt. Comm., vol 39, pp 211-215, 1981.

22. See Chap. 12 and 13 in 13.

23. P. Hello, "Optical aspects of interferometric gravitational-wave detectors", Progress in Optics, vol. XXXVIII, pp. 85-164, 1998.

24. J.A. Arnaud, "Nonorthogonal waveguides and resonators", Bell Syst. Tech. J., pp. 2311-2348, 1970.

25. A.G. Fox and T. Li, "Resonant modes in a maser interferometer", Bell Syst. Tech. J., vol. 40, pp. 453-488, 1960.

26. R.L. Sanderson and W. Streifer, "Comparison of laser mode calculations", Appl. Opt., vol. 8, pp.131-136, 1969.

27. A.E. Siegman and H.Y. Miller, "Unstable optical resonator loss calculations using the Prony method", Appl. Opt., vol.9, pp.2729-2736, 1970.

28. M.D. Feit, J.A. Fleck, "Spectral approach to optical resonator theory", Appl. Opt., vol. 20, pp. 2843-2851, 1981.

29. K. Altmann, C. Pflaum and D. Seider, "Three-dimensional finite element computation of laser cavity eigenmodes", Appl. Opt., vol. 43, pp. 1892-1901, 2004.

30. C.J. Hood, H.J. Kimble and J. Ye, "Characterization of high-finesse mirrors: loss, phase shifts, and mode structure in optical cavity", Phys. Rev. A, vol. 64, pp. 033804-033811, 2001.

31. A. Ciattoni, B. Crosignani and P. Di Porto, "Vectorial free-space optical propagation: a simple approach for generating all-order nonparaxial corrections", Opt. Comm., vol. 177, pp. 9-13, 2000.

32. Maple 9 software program, Waterloo Maple Inc., 57 Erb Street West, Waterloo, Ontario, Canada.

33. Matlab 6.5 software, The MathWorks Inc., 3 Apple Hill Drive, Natick, United States of America.

34. W.H. Carter, "Electromagnetic field of a Gaussian beam with elliptical cross section", J. Opt Soc. Am., vol.62, pp. 1195-1201, 1972.

35. J.P. Gordon and H. Kogelnik, "Equivalence relations among spherical mirror optical resonators", Bell Syst. Tech. J., vol 43, pp 2873-2886, 1964.

36. C.J.R Sheppard and P. Török, "Dependence of focal shift on Fresnel number and angular aperture", Opt. Lett., vol 23, pp 1803-1804, 1998.

37. L.A. Vainshtein, “Open resonators for lasers”, Sov. Phys. JETP, vol 17, pp 709-719, 1963.

38. A.E. Siegman, Lasers, CA: University Science Books, Sausalito 1986, p 770.

39. S. Riyopoulos, D. Dialetis, J. Inman and A. Phillips, "Active-cavity vertical-cavity surfaceemitting laser eigenmodes with simple analytic representation", J. Opt. Soc. Am. B, vol. 18, pp. 1268-1284, 2001.

40. C.J.R. Sheppard and S. Saghafi, "Beam modes beyond the paraxial approximation: a scalar treatment”, Phys. Rev. A, vol 57, pp 2971-2979, 1998. 
41. C.J.R. Sheppard and S. Saghafi, "Electromagnetic Gaussian beams beyond the paraxial approximation”, J. Opt. Soc. Am. A, vol 16, pp 1381-1386, 1999.

42. D.H. Foster and J.U. Nöckel, "Spatial and polarization structure in micro-dome resonators: effects of a Bragg mirror", Proc. SPIE, vol 5333, pp 195-203, 2004. 